\title{
CONSEQUENCES A LONG TERME DE LA VASECTOMIE : REVUE DE LA LITTERATURE
}

\author{
Patrick Thonneau et Béatrice D'Isle \\ Centre de Contraception (consultation Vasectomie) de l'hôpital Louis-Mourier \\ 178 , rue des Renouillers - 92700 COLOMBES.
}

\begin{abstract}
LONG TERM EFFECTS OF VASEC. TOMY : REVIEW OF THE LITTERATURE. Since 20 years, many contradictory data have been reported about vasectomy in animals and in humans, as regards its efficacy and safety. We have examined the main experimental and clinical reports, published these last 20 years. In particular experimental conditions, in animals subject to hypercholesterolemic diets, vasectomy would cause negative effects, particularly an increased atheroma risk. But, none of the large epidemiologic inquiries performed until now in several developed and on developing countries, showed an increased vascular and infarction risk in vasectomized males. Key-words : male sterilization, vasectomy, reproduction epidemiology.

Andrologie : 1991, $1: 26-28$
\end{abstract}

Au cours des dix dernières années, de nombreuses études sur les effets de la vasectomie ont été réalisées chez l'animal et chez l'homme. Les résultats souvent contradictoires de ces travaux et les controverses qui s'en sont suivies ont entraîné des suspicions vis-à-vis de l'acceptabilité générale de cette méthode.

John Hunter en 1775, puis Sir Astley COOPER en 1830 mentionnèrent les premiers la vasectomie. Au début de ce siècle, la vasectomie fut utilisée comme substitut de castration dans le traitement des affections prostatiques, puis cette technique connut un premier essor aux EtatsUnis, dans les premières années du $\mathrm{XX}^{\mathrm{e}}$ siècle, pour la stérilisation des débiles mentaux et des criminels. Considérée à partir de 1968 comme "une méthode de planification familiale à part entière" par l'American Medical Association, la vasectomie s'est rapidement développée dans les pays anglo-saxons et dans certains pays en développement.

Adapté de l'article des mêmes auteurs "Review : Does vasectomy have long-term effects on somatic and psychological health status ?", paru dans Intemational Joumal of Andrology, 1990, 13:419-432

\section{ASPECTS SOMATIQUES \\ Conséquences hormonales}

Les premiers travaux sur les conséquences endocriniennes de la vasectomie ont été effectués chez le rat par Steinach en 1927 (30), qui suggérait que la vasectomie puisse entraîner une augmentation de la production de testostérone.

Bunge (1972) (6), puis Tyler (1979) en utilisant les premiers dosages radio-immunologiques, n'observèrent aucune conséquence hormonale chez les hommes vasectomisés. Goebelsmann, 1979 (13), De la Torre, 1983 (9), Peng, 1987 (24) ont comparé chez des hommes vasectomisés et des hommes témoins non-vasectomisés, les taux de LH, FSH, DHT.S et prolactine sans retrouver de différence significative entre les sujets vasectomisés et les témoins.

Conséquences sur les glandes annexes Afin d'apprécier le retentissement éventuel de la vasectomie sur la fonction prostatique Marmar (19) a comparé , en 1975, les taux de zinc chez trois groupes d'hommes (hommes volontaires sains, vasectomisés, présentant une prostatite) sans trouver de différence entre le groupe d'hommes vasectomisés et celui des volontaires sains.

Toutefois Naik (22), dans une étude comparative faite en 1980 entre 78 hommes vasectomisés et 22 hommes féconds, notait une baisse significative de tous les constituants du plasma séminal après vasectomie. Parrish (23) a comparé en 1987 la concentration séminale du zinc parmi quatre groupes d'hommes (nonvasectomisés, récemment vasectomisés, vasectomisés depuis plusieurs années, vasovasostomisés). Ses résultats ont confirmé les travaux de Marmar et l'absence d'effet de la vasectomie sur le taux de zinc séminal.

\section{ASPECTS IMMUNOLOGIQUES CHEZ L'ANIMAL ET CHEZ L'HOMME}

Après une vasectomie, les antigènes spermatozoïdaux (Ags) vont rapidement entraîner une réaction immunitaire avec production d'anticorps anti-spermatozoïdes (Acps) : après un an des Acps agglutinants sont trouvés chez 40 à $70 \%$ des hommes, des Acps immobilisants chez 25 à $60 \%$. La plupart des recherches réalisées chez l'animal et chez l'homme, à propos de la vasectomie, ont concerné ces réactions immunologiques, en essayant d'en évaluer les conséquences.

\section{Chez l'animal}

En 1978 et en 1980, Alexander (3) et Clarkson (8) publiaient les premiers articles concernant le retentissement de la vasectomie sur les phénomènes athéromateux. Des singes vasectomisés soumis à un régime hypercholestérolémiant, comparés à des singes témoins (non-vasectomisés et soumis au même régime), présentaient une augmentation significative dans la rapidité et l'étendue des lésions coronariennes athéromateuses. Les auteurs évoquaient le rôle possible des immuns complexes circulants (conséquences de la combinaison Ags-Acps) avec dépots au niveau des parois artérielles et création de plaques athéromateuses. Des résultats semblables furent retrouvés chez de nombreuses espèces animales : atteintes glomérulaires chez les lapins vasectomisés, chez le cochon et chez le singe macaque où l'injection de fragments cellulaires émanant d'animaux vasectomisés entrainait des orchites auto-immunes. Toutefois Lauersen, en 1983 (17), reprenant l'expérience d'Alexander chez des singes vasectomisés soumis à un régime hypercholestérolémiant, ne retrouve aucune différence significative entre les deux groupes de singes, vasectomisés et nonvasectomisés.

Comme le font remarquer Bedford (1976) (4), puis Linnet (1983) (18), les résultats assez inquiétants de ces travaux expérimentaux, réalisés chez des espèces animales très différentes et soumises à des conditions alimentaires particulières, doivent être considérés avec prudence et circonspection quant à leur extrapolation directe à l'homme.

\section{Chez l'homme \\ Vasectomie et cancer}

En 1987 et 1988, Thornhill, $(31,32)$ examinant les registres des cancers survenus en Irlande de 1980 à 1985 , notait un nombre anormalement élevé de tumeurs testiculaires chez les hommes vasectomisés. Tout en précisant que les tumeurs devaient préexister avant la vasectomie, les auteurs évoquaient le rôle possible de la vasectomie en tant que phénomène accélérateur d'un processus néoplasique préalablement existant. Cale (7), en Ecosse (1990), retrouve des 
résultats analogues parmi les patients hospitalisés au cours des dix dernières années dans un hôpital général. Toutefois, le faible nombre d'hommes vasectomisés présentant une tumeur testiculaire (8 cas), leur concentration dans la classe d'âge $30-35$ ans (6/8) et la découverte de la tumeur testiculaire dans les deux années suivant la vasectomie, doivent susciter beaucoup de prudence dans les interprétations statistiques et inciter à des recherches ultérieures sur ce sujet.

Vasectomie et lésions athéromateuses En 1980, Farhenbach (12) trouvait des lésions rétiniennes chez les hommes vasectomisés et suggérait le rôle immunologique de la vasectomie comme l'hypothèse physiopathologique de ces troubles; ces résultats n'ont pas été retrouvés dans une étude similaire (18).

Alexander, 1981 (1), en étudiant les variations de la tension artérielle chez 946 hommes donneurs de sang dont $30 \%$ vasectomisés, a trouvé une légère augmentation (mais non statistiquement significative) de la pression artérielle systolique chez les sujets vasectomisés. En 1986, Alexander (2) a comparé un groupe de 101 hommes vasectomisés à un groupe de 101 hommes témoins non-vasectomisés (avec, à l'intérieur de chaque groupe, 31 hommes ayant eu des antécédents coronariens) et notait une augmentation significative des immuns complexes circulants chez les sujets vasectomisés.

\section{Vasectomie et grandes enquêtes épidémiologiques}

Walker (34), en utilisant les données médicales du Group Health Cooperative of Puget Sound à Seattle a comparé en 1981 et en 1983 un groupe de 4733 hommes vasectomisés à cinq groupes témoins. Plus de 10 ans en moyenne après la vasectomie, l'incidence d'infarctus du myocarde était identique entre les groupes. Les motifs et les taux d'hospitalisations étaient identiques entre les groupes, sauf pour les pathologies génitourinaires pour lesquelles les hommes vasectomisés présentaient, au cours des premières années, une incidence plus élevée (une partie de cette majoration pouvant s'expliquer par la prise en compte dans ces taux des incidents immédiats post-vasectomies).

Petiti $(1982,1983)(27)$ en utilisant les questionnaires médicaux du Programme de soins médicaux de la Société Northern California Kaiser Permanente (1,8 millions d'adhérents) a comparé 4385 hommes vasectomisés à 13135 hommes non vasectomisés (appariés selon l'âge et la race). Les taux d'hospitalisation pour les 16 maladies retenues dans cette étude, dont les pathologies cardio-vasculaires et ischémiques, étaient identiques dans les deux groupes (aucune différence n'était notée selon le délai écoulé depuis la vasectomie, que celui-ci ait été ou non supérieur à dix années).

Chez 10632 hommes inclus dans une enquête sur les facteurs de risque coronarien (University of Washington Exercise Testing Registry) Perrin, en 1984, (25), a montré que si le risque coronarien était associé à la présence d'antécédents coronariens familiaux, d'hypertension artérielle ou de tabagisme, il n'était pas majoré par le fait d'avoir eu une vasectomie (et ceci quel que soit le temps écoulé depuis l'intervention).

Massey, 1984 (20) a constitué, aux Etats-Unis, la plus importante cohorte multicentrique (Mineapolis, Rochester, Los Angeles, Eureka) comprenant 10590 hommes vasectomisés comparés à 21.180 témoins (la durée écoulée entre l'entrevue pour cette enquête et la vasectomie variait de 1 à 42 ans, avec une moyenne de 7,9 années, 2318 hommes ayant eu leur vasectomie depuis plus de 10 ans). Excepté pour les orchi-épididymites, l'incidence des pathologies cardio-vasculaires, hypertensives, néoplasiques, digestives ou psychiatriques était, chez les hommes vasectomisés, égale voire inférieure à celle des sujets témoins nonvasectomisés. Une étude sismilaire (14) effectuée dans huit communes rurales de la région de Sechouan en Chine a montré que le risque de décès était moindre chez les hommes vasectomisés comparés aux hommes nonvasectomisés et que les hommes vasectomisés étaient, au moment de l'enquête, en meilleure santé que les hommes témoins.

En termes de santé publique, les résultats de ces études épidémiologiques confirment l'innocuité à long terme de la vasectomie sur la santé physique des individus. Les contradictions entre les études cliniques et les enquêtes épidémiologiques pourraient résulter de certaines conditions expérimentales ou méthodologiques (ainsi l'effet de sélection pourrait conduire les individus se sachant en mauvaise santé a ne pas solliciter une vasectomie, et les praticiens à récuser les hommes en mauvaise santé ou présentant des facteurs de risque à long terme (comme c'est le cas pour les troubles psychologiques qui constituent une contre-indication relative à la pratique de la vasectomie).

\section{ASPECTS PSYCHOLOGIQUES}

Plus de $90 \%$ des hommes vasectomisés se disent satisfaits d'avoir fait faire cette intervention, ce qui ne signifie pas pour autant qu'il n'existe aucun problème (5).

La vasectomie n'entraîne pas de changement notable dans la vie familiale et sexuelle de l'homme vasectomisé (10).Lorsque les hommes n'expriment pas de satisfaction après l'intervention demandée, les différents auteurs parlent de "regret", "mécontentement", "insatisfaction". Toutefois ces trois termes doivent être pris de façon nuancée, car les critères de ces auteurs sont éminemment variables. En tous cas, on ne peut pas se fier au pourcentage des hommes qui demandent une réanastomose pour apprécier efficacement les conséquences négatives de la vasectomie : Ne pas demander de réanastomose ne signifie pas pour autant que l'on n'a pas de regrets...

Les répercussions de la vasectomie sur la santé mentale des hommes vasectomisés sont une préoccupation constante des praticiens $(15,28,35)$. A l'heure actuelle, il est à peu près certain que la vasectomie n'a aucune influence sur la santé mentale. Par contre, il importe d'être attentif à la question de la santé mentale du demandeur avant la vasectomie. Car ce sont plutôt les sujets peu sûrs de leur décision qui risqueraient ensuite de souffrir d'éventuelles conséquences négatives. La vasectomie n'est pas une chirurgie d'urgence et nécessite qu'on prenne tout son temps avant d'intervenir. (21) Les études $(29,36)$ sur les "attitudes" démontrent au fond que la stérilisation est d'autant mieux considérée et choisie que les autres alternatives sont envisagées négativement. Il semble que la peur d'avoir un enfant non désiré soit un bon facteur pronostique de la vasectomie!

A l'issue de cette recherche bibliographique aucun effet secondaire majeur, physique ou psychologique n'a été retrouvé, à ce jour, chez les hommes vasectomisés. Sur le plan de l'innocuité et de l'efficacité, la vasectomie se compare favorablement à la ligature de trompes (avec pour la ligature : risque de morbidité et de mortalité non nul, durée d'hospitalisation et coût plus élevé, taux d'échecs nettement supérieur). Il faut souhaiter que la vasectomie puisse obtenir, outre un véritable statut juridique qu'elle n'a toujours pas en France (11) un "droit de cité médical" pour les couples souhaitant réguler leur vie reproductive.

\section{REFERENCES}

1 - Alexander N.J., Senner J.W., Hoch E.J. Evaluation of blood pressure in vasectomized and nonvasectomized men. International Journal of Epidemiology, 1981, $10: 217-222$.

2 - Alexander N.J., Fulgham D.L., Plunkett E.R., Witkin S.S. Antisperm antibodies and circulating immune complexes of vasectomized men are without coronary events. American Journal Reproductive Immunology and Microbiology, 1986, $12: 38-44$.

3 - Alexander N.J., Clarkson T.B. Vasectomy increases the severity of diet-induced atherosclerosis in Macaca fascicularis. Science, 1978, 201 : 538-541.

4 - Bedford J.M. Adaptations of the male reproductive tract and the fate of spermatozoa following vasectomy in the rabbit, rhesus monkey, hamster and rat. Biology and Reproduction, 1976, 14:219-221.

5 - Bourget F., Bourmeau A., Le Danois A. L'expérience nantaise de la vasectomie : analyse d'une série de 272 vasectomies. Les suites médicales et 
psycho-sexuelles. Contraception Fertilité Sexualité, 1983, 11; 435-438.

6 - Bunge R.G., Plasma testosterone levels in man before and after vasectomy. Investigation in Urology, 1972, 10:9-11.

7 - Cale ARJ, Farouk M., Prescott RJ., Wallace IWJ : Does vasectomy accelerate testicular tumour? Importance of testicular examinations before and after vasectomy. British Medical Journal 1990, 300: 370-370. 8 - Clarkson T.B., Alexander N.J. Long-term vasectomy : effects on the occurrence and extent of atherosclerosis in rhesus monkey. Journal of Clinical Investigation, 1980, $65: 15-25$.

9 - De la Torre B., Hedman M., Jensen F., Pedersen P.H., Diczfalusy E. Lack of effect of vasectomy on peripheral ganadotrophin and steroid levels. International Journal of Andrology 1983, $6: 125-34$.

10 - Dias P.L.R. The effects of vasectomy on testicular volume. British Journal of Urology, 1983, 55 : 83-84.

11 - Dourlen-Rollier A.M. La vasectomie : libéralisation en Italie, immobilisme en France. ContraceptionFertilité-Sexualité, 1988, $16: 252-254$.

12 - Fahrenbach H.B., Alexander N.J., Senner J.W., Fulgham DL., Coon LJ. Effect of vasectomy on the retinal vasculature of men. Journal of Andrology, 1980, $1: 299-303$.

13 - Goebelsmann U., Bernstein G.S., Gale J.A., Kletzky O.A., Nakamura R.M., Coulson A.H. \& Korelitz J.J. Serum gonadotropin, testosterone, oestradiol and oestrone levels prior to and following bilateral vasectomy, in Lepow I.H et Crozier R. (Eds) : Vasectomy : Immunologic and pathophysiologic effects in animals and man. New-York, Academic Press 1979,165-181.

14 - Guang-Hua T., Yu-Hui Z., Yue-Min M., Lin L. al. Vasectomy and Health : Cardiovascular and Other Diseases Following Vasectomy in Sichuan Province, People's Republic of China. International Journal of Epidemiology 1988, 17: 608-17.

15 - Jouannet P. Qui demande la vasectomie en France? Les paternités impossibles, volontaires ou refusées. Les pères aujourd'hui- Bulletin de l'Institut National d'Etudes Démographiques 1982: 81-85.

16 Kronmal R.A., Krieger J.N Kenney J.W., Alderman E., Killip T., Athearn M. Vasectomy and urolithiasis. Lancet, 1988, 2 janvier : 22-23.

17 - Lauersen N.H., Muchmore E., Shulman S., Marinescu A.E., Graves Z.R., Leeds L., Geller S.A. Vasectomy and atherosclerosis in Macaca fascicularis : new findings in a controversial issue. Journal of
Reproduction and Medecine, 1983, 28 : 750-758.

18 - Linnet L., Moller N.P.H., Bernth-Petersen P., Ehlers N., Brandslund I., Svehag S. No increase in arteriolosclerotic retinopathy or activity in tests for circulating immune complexes 5 years after vasectomy. Fertility and Sterility, 1982, 37 : 798-806.

19 - Marmar J.L. ,Katz S., Praiss D.E. \& De Benedictis T. Semen zinc levels in infertile and postvasectomy patients and with prostatitis. Fertility and Sterility, 1975, 26: 1057-1063.

20 - Massey F.J., Bernstein G.S., O'Fallon W.M., Schuman L.M., Coulson A.H., Crozier R., Mandel J.S., Benjamin R.B., Berendes H.W., Chang P.C. et al. Vasectomy and health : results from a large cohort study. Journal of the American Medical Association, 1984, 252: 1023-1029.

21 - Mumford S.D.. The vasectomy decision-making process. Studies in Family Planning, 1983, 14: 83-88.

22 - Naik V.K., Joshi U.M., Sheth A.R. Long term effects of vasectomy on prostatic function in men. Joumal of Reproduction and Fertility, 1980, $58: 289$. 293.23 - Parrish R.F., Kessler R., Shapiro C.E., Fair W.R. Vasectomy and vaso-vasostomy have no effect on seminal plasma zinc concentrations. Journal of Urology, 1987, 137: 228-229.

24 - Peng X.S., Li F.D., Miao Z.R., Ye X.M., Wong Y., Hu X.Z., Zhong Z.Z., Zeng F.X., Wu X.Q., Lan J., Li G.Z., Jing S.S., Farley T.M.M. Plasma reproductive hormones in normal and vasectomized Chinese males. International Journal of Andrology, 1978, 10:471-479.

25 - Perrin E.B., Woods J.S., Namekata T., Yagi J., Bruce R.A., Hofer V. Long-term effect of vasectomy on coronary heart disease. American Journal of Public Health, 1984, 74: 128-132.

26 - Petitti D.B., Klein R., Kipp H., Kahn W., Siegelaub A.B., Friedman G.D. A survey of personal habits, symptoms of illness, and histories of disease in men with and without vasectomies. American Journal of Public Health, 1982, 72: 476-480.

27 - Petitti D.B., Klein R.,Kipp H., Friedman G.D. Vasectomy and the incidence of hospitalized illness. Journal of Urology, 1983, $129: 760-762$.

28 Philliber S.G., Philliber W.W. Social and psychological perspectives in voluntary sterilization : A review. Studies in Family Planning, 1985, 16: 1-29.

29 - Santiso R., Bertrand J.T., Pineda M.A., Guerra S. Public opinion on and potential demand for vasectomy in semi-rural Guatemala. American Journal of Public Health, 1985, 75:73-75.

30 - Steinach E. Biological method against the process of old age. Medical Journal for Research, 1927, $125: 77$.

31 - Thornhill J.A., Butler M., Fitzpatrick J.M. Could vasectomy accelerate testicular cancer? The importance of pre-vasectomy examination. Britisi Journal of Urology, 1987, $59: 367$.

32 - Thomhill J.A., Conroy R.M., Kelly D.G.,Walsh A., Fennelly J.J., Fitzpatrick J.M. An evaluation of predisposing factors for testis cancer in Ireland. European Urology, 1988, $14: 429-433$.

33 - Tyler J.P.P., Richardson D.W., Newton J.R. The hormonal and immunological status of vasectomized men. Contraception, 1979, $19: 599-611$.

34 - Walker A.M., Jick H., Hunter J.R., \& McEvoy J. Vasectomy and nonfatal infarction : continued observation indicates no elevation of risk. Journal of Urology, 1983, $130:$ 936-937.

35 - Williams D., Swicegood G., Clark M.P., Bean F.D. Masculinity - Feminity and the desire for sexual intercourse after vasectomy : A longitudinal study. Social Psychological Quaterly, 1980, 43, 3: 347-352.

36 - Ziegler F.J., Rodgers D.A. \& Prentis R.J. Psychosocial response to vasectomy. Archives

RESUME : Depuis une vingtaine d'années, la vasectomie a fait l'objet de nombreux travaux contradictoires, chez l'animal et chez l'homme, quant à son efficacité et son innocuité. A partir d'une revue exhaustive de la littérature des vingt dernières années, nous avons examiné les principaux travaux, expérimentaux et cliniques, ayant eu lieu dans ce domaine. Dans des conditions expérimentales particulières, chez les animaux soumis à des régimes hypercholestérolémiants, la vasectomie produirait des conséquences néfastes et en particulier une majoration du risque athéromateux. Cela étant, aucune des grandes enquêtes épidémiologiques réalisées à ce jour dans plusieurs pays développés et en développement, n'a montré une quelconque majoration du risque cardio-vasculaire ou coronarien chez les hommes vasectomisés. Mots clés : Stérilisation masculine, vasectomie, epidémiologie de la reproduction. Andrologie, 1991, 1 : 26-28 